\title{
Effects of in-Scanner Bilateral Frontal tDCS on Functional Connectivity of the Working Memory Network in Older Adults
}

\begin{abstract}
Nicole R. Nissim ${ }^{1,2}$, Andrew O'Shea ${ }^{1}$, Aprinda Indahlastari ${ }^{1}$, Rachel Telles ${ }^{1}$, Lindsey Richards ${ }^{1}$, Eric Porges ${ }^{1}$, Ronald Cohen ${ }^{1}$ and Adam J. Woods ${ }^{1,2 *}$
\end{abstract}

${ }^{1}$ Center for Cognitive Aging and Memory, Department of Clinical and Health Psychology, McKnight Brain Institute, University of Florida, Gainesville, FL, United States, ${ }^{2}$ Department of Neuroscience, University of Florida, Gainesville, FL, United States

Working memory is an executive memory process essential for everyday decisionmaking and problem solving that declines with advanced age. Transcranial direct current stimulation (tDCS) is a non-invasive form of brain stimulation that has demonstrated potential for improving working memory performance in older adults. However, the neural mechanisms underlying effects of tDCS on working memory are not well understood. This mechanistic study investigated the acute and after-effects of bilateral frontal (F3/F4) tDCS at $2 \mathrm{~mA}$ for 12-min on functional connectivity of the working memory network in older adults. We hypothesized active tDCS over sham would increase frontal connectivity during working memory performance. The study used

OPEN ACCESS

Edited by:

P. Hemachandra Reddy, Texas Tech University Health Sciences Center, United States

Reviewed by: Milton Cesar Biagioni, New York University, United States Rongqiao He, Institute of Biophysics (CAS), China

${ }^{*}$ Correspondence: Adam J. Woods ajwoods@ufl.edu

Received: 19 December 2018 Accepted: 22 February 2019 Published: 15 March 2019

Citation: Nissim NR, O'Shea A, Indahlastari A, Telles R, Richards L, Porges $E$, Cohen $R$ and Woods AJ (2019) Effects of in-Scanner Bilateral Frontal tDCS on Functional Connectivity of the Working Memory Network in Older Adults. Front. Aging Neurosci. 11:51. doi: 10.3389/fnagi.2019.00051 a double-blind within-subject 2 session crossover design. Participants performed an functional magnetic resonance imaging (fMRI) N-Back working memory task before, during, and after active or sham stimulation. Functional connectivity of the working memory network was assessed within and between stimulation conditions $($ FDR $<0.05)$. Active tDCS produced a significant increase in functional connectivity between left ventrolateral prefrontal cortex (VLPFC) and left dorsolateral PFC (DLPFC) during stimulation, but not after stimulation. Connectivity did not significantly increase with sham stimulation. In addition, our data demonstrated both state-dependent and time-dependent effects of tDCS working memory network connectivity in older adults. tDCS during working memory performance produces a selective change in functional connectivity of the working memory network in older adults. These data provide important mechanistic insight into the effects of tDCS on brain connectivity in older adults, as well as key methodological considerations for tDCS-working memory studies.

Keywords: tDCS, fMRI-functional magnetic resonance imaging, cognitive aging, DLPFC (dorsolateral prefrontal cortex), working memory, transcranial direct cortical stimulation (tDCS), functional connectivity

\section{INTRODUCTION}

Working memory enables us to remember, manipulate, and reorganize information for short periods of time (Salthouse et al., 1989; Baddeley, 1992, 2003). This executive memory process is fundamental for everyday life and is known to decline with advanced age (Li et al., 2001; Gazzaley et al., 2007). Age-related decline in working memory significantly contributes 
to loss of independence and decreased quality of life (Mograbi et al., 2014). However, there is currently a paucity of effective interventions for remediating working memory decline in older adults. Transcranial direct current stimulation (tDCS) demonstrates promise as a potential intervention in this domain, but knowledge of the mechanisms underlying tDCS effects on working memory function in older adults remains unexplored.

Non-human primate studies have indicated the importance of the frontal lobes, particularly the dorsolateral prefrontal cortex (DLPFC), in working memory function (Goldman-Rakic, 1987, 1996; Wang et al., 2011). In humans, structural and functional neuroimaging studies support the integral importance of frontal structures in working memory (Boisgueheneuc et al., 2006; Barbey et al., 2013). Reduced structural surface area of right frontal lobe regions in older adults is associated with lower working memory performance (Nissim et al., 2017). In addition, low performing older adults demonstrate significantly increased right lateralized blood oxygenation level dependent (BOLD) response compared to high performing older adults who demonstrate bilateral recruitment of frontal structures (i.e., neural compensation hypothesis; Cabeza et al., 2002). Moreover, functional connectivity of the working memory network decreases with older age (Hampson et al., 2006; Andrews-Hanna et al., 2007; Hampson et al., 2010; Keller et al., 2015). While there is growing evidence supporting the role of frontal structural and functional decline in age-related working memory performance, there is a lack of effective interventions aimed at treating this decline. Such interventions will be necessary for addressing public health concerns related to the increasing age of the world population and the increased prevalence of age-related cognitive decline in the population at large.

Non-invasive brain stimulation techniques have the potential to impact cognitive processes and modify behavior by inducing changes in brain function (Gomes-Osman et al., 2018). tDCS is a safe and painless form of non-invasive brain stimulation that modulates the neuroplastic response of brain tissue and can impact behavior both during and after periods of stimulation through sub-threshold alteration of resting membrane potentials (Nitsche and Paulus, 2000; Monte-Silva et al., 2013; Pelletier and Cicchetti, 2015; Bikson et al., 2016; Woods et al., 2016; Bikson et al., 2018; Knotkova et al., 2019). Prior studies have demonstrated that tDCS can impact and enhance working memory in older adults (Jones et al., 2015; Woods et al., 2019a). For example, Stephens and Berryhill (2016) demonstrated that older adults who received active tDCS over sham (anode-F4, cathode-contralateral cheek) during a working memory training protocol experienced greater benefits on untrained tasks at post-training assessment (Stephens and Berryhill, 2016). Effects of tDCS combined with working memory training have shown to extend and increase training gains (Richmond et al., 2011, 2014; Jones et al., 2015; Stephens and Berryhill, 2016). Recent research also suggests that the efficacy and transfer of tDCS paired with working memory training may be state dependent, with greater transfer occurring when training tasks are more difficult (Gill et al., 2014). These studies demonstrate the promise of tDCS as a potential intervention for age-related working memory decline.

However, there is a gap in knowledge on the mechanism(s) of action of tDCS-based enhancement of working memory in the brain. No study to date has directly investigated the impact of tDCS on functional connectivity of the working memory network. Better understanding of the mechanism(s) of action for tDCS-based working memory enhancement is essential to harness and optimize tDCS as a treatment approach for cognitive decline in older adults. To address this gap in knowledge, we performed a mechanistic study designed to evaluate the acute and after-effects of tDCS on functional connectivity of working memory networks in older adults during N-Back performance (2- vs. 0-back). This study specifically aimed to query the direct impact of tDCS vs. sham on the working memory network, taking steps to stabilize test-retest behavioral and network change across N-back sessions and between conditions (active vs. sham) to focus our inquiry on direct mechanistic effects of an acute tDCS dose on the network. We hypothesized that active stimulation vs. sham would result in increased frontal connectivity during working memory performance in older adults. In addition, we hypothesized that changes in functional connectivity from active tDCS would be greater in the high-effort 2-Back vs. 0-Back conditions.

\section{MATERIALS AND METHODS}

We conducted a randomized double-blinded crossover withinsubject study examining the acute effects of bilateral frontal tDCS (2 mA for $12 \mathrm{~min}$; F3/F4) during an functional magnetic resonance imaging (fMRI) BOLD scan. For each stimulation condition (active vs. sham), participants performed three runs (baseline-active/sham, during-active/sham, post-active/sham) of an N-Back working memory task. Participants received each stimulation condition (active and sham) at two separate visits (randomized and counterbalanced), and thus, served as their own controls. Figure 1 illustrates the overall experimental design used in this study.

\section{Participants}

Sixteen older adults between the ages of 61-82 years old participated in this study $[\mathrm{n}$ female $=6$; mean age $(\mathrm{SD})=71.75$ (7.29); mean education $=16.8(1.92)]$. All participants completed a medical history questionnaire and cognitive assessments to meet study eligibility criteria. The Montreal Cognitive Assessment (MoCA) was used to screen for significant global cognitive deficits. Participants were enrolled in the study if they were MRI-compatible, able to receive electrical stimulation on the head, scored at least 20 or more on the MoCA (mean $=26.56 \mathrm{SD}=2.94)$, were right-hand dominant, and not taking certain medications potentially blocking tDCS excitability effects (i.e., GABAergic, glutamatergic, or sodium channel blockers; McLaren et al., 2018). Participants with a history of neurological disorders, seizures, psychiatric disorders, in active treatment for cancer, previous traumatic brain injury with loss of consciousness greater than $20 \mathrm{~min}$, or any neurodegenerative disease were excluded from the study. The 


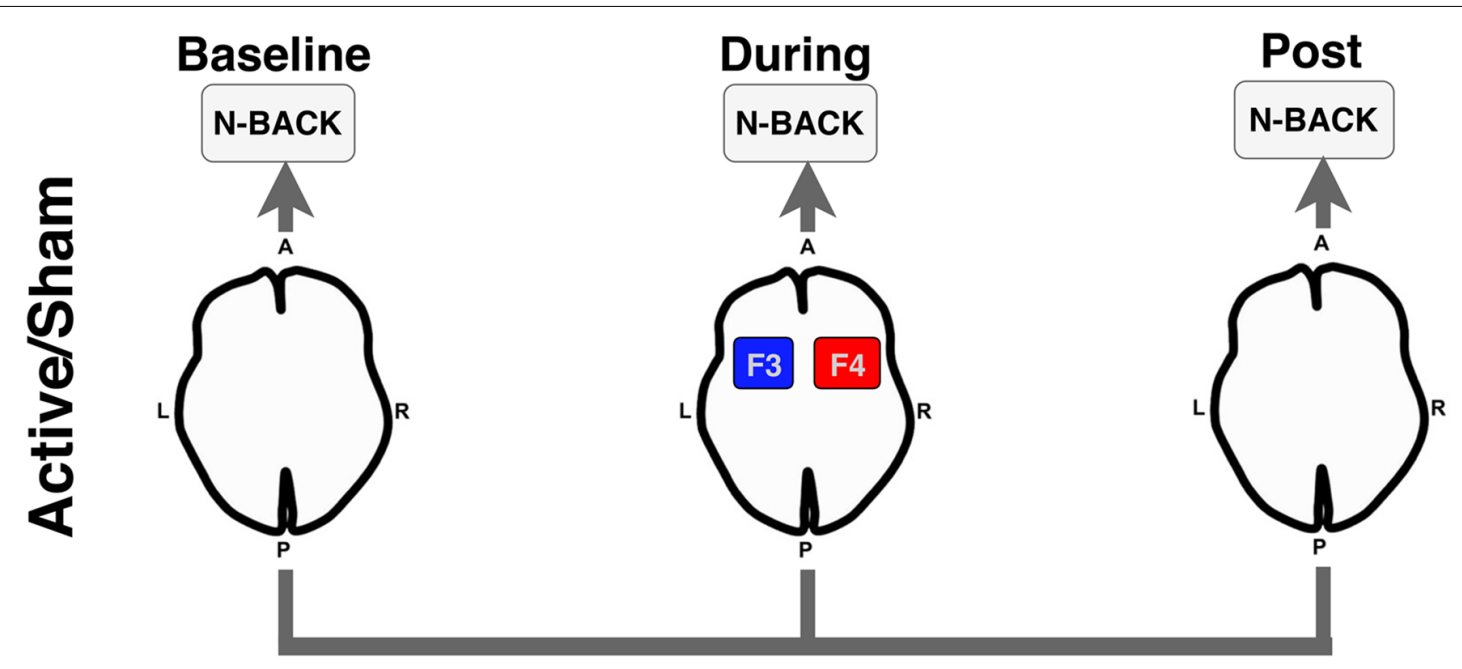

FIGURE 1 | Experimental design for the functional magnetic resonance imaging (fMRI) N-Back task. Participants underwent an N-Back task before (Baseline), during active or sham stimulation (During), and after stimulation was stopped (Post). Anode = red; Cathode = blue. A, anterior; P, posterior; L, left; R, right.

washout period between stimulation visits for each participant was 8.5 days on average (range $=6-16$ days). The study protocol was in accordance with the Declaration of Helsinki and approved by University of Florida's Institutional Review Board. Participants provided written informed consent prior to any study procedures.

\section{Structural MRI Acquisition}

T1-weighted MPRAGE structural MRI scans were obtained prior to BOLD fMRI using a 32-channel head coil (3T Philips $\mathrm{MRI})$. Scan parameters included: repetition time $(\mathrm{TR})=7.0 \mathrm{~ms}$; echo time (TE $=3.2 \mathrm{~ms}$ ); flip angle $=8^{\circ}$; field of view $($ FOV $)=240 \times 240 \times 170 \mathrm{~mm}$; voxel size $=1 \mathrm{~mm}^{3}$.

\section{fMRI Data Acquisition}

fMRI scans were acquired on a 3 Tesla Philips MRI scanner using a 32-channel head coil. Task-based fMRI data were collected using a single-shot EPI sequence (36 slices, no gap, $\mathrm{TR}=2,000 \mathrm{~ms}, \mathrm{TE}=30 \mathrm{~ms}$, flip angle $=80^{\circ}$, FOV $=224 \times 224 \times 126$, voxel size $=3.5 \mathrm{~mm}^{3}$ ). Task stimuli were presented on a screen and reflected onto a mirror visible to participants. Participants were trained on the task prior to the MRI session.

\section{tDCS Parameters and Application}

Bilateral frontal tDCS was delivered inside the scanner at $2 \mathrm{~mA}$ for $12 \mathrm{~min}$ (30 $\mathrm{s} \mathrm{ramp}$ up/down) during the active condition using an MRI-compatible tDCS device (neuroConn DC-Stimulator MR). The sham condition was identical except that the stimulation period lasted only $30 \mathrm{~s}$. Prior studies that examined $1 \mathrm{~mA}$ intensity consistently report increased excitation under the anode and decreased excitation under the cathode electrode in the context of motor-evoked potential TMS paradigms in the motor cortex (Nitsche and Paulus, 2000; Nitsche et al., 2003a,b, 2004). In contrast, increasing the intensity to $2 \mathrm{~mA}$ has been shown to produce net excitation under both anode and cathode electrodes (Batsikadze et al., 2013; Reato et al., 2019). Our montage (F3/F4) and parameter selection of $2 \mathrm{~mA}$ was chosen to broadly target bilateral frontal brain regions involved in working memory with the intent of producing net excitation under the anode and cathode during active stimulation. Head measurements were performed using the 10-20 International EEG System to identify F3 (cathode) and F4 (anode) locations over left and right DLPFC, respectively. A $5 \mathrm{~mm}$ thick electrical conductive paste (Ten20 paste) was applied directly on two $5 \times 7 \mathrm{~cm}^{2}$ rubber electrodes prior to placing the electrodes onto the scalp before the scan (Khadka et al., 2019; Woods et al., 2019b). A thin layer of paste was also applied to the scalp at the F3/F4 locations for at least 30 min before the scan to allow paste to saturate the skin/scalp and achieve target impedance levels ( $<2 \mathrm{kOhms})$. The electrodes were held in place with a rubber Soterix Easy-Head strap. The device was turned on before the start of the second run of the N-Back to allow ramp time to complete prior to start of the fMRI scan. A blinded six-digit code was entered into the device to initiate the randomized stimulation condition for the session. No adverse events were reported during the study.

\section{N-Back Task}

During the task period (36 min; 12 min per run), participants performed three runs of the N-Back task (baseline, during, post) for each stimulation condition (active and sham). The task paradigm for each run consisted of four blocks of 2-Back and four blocks of 0 -Back presented in a randomized order. In total, 12 blocks of both 2-Back and 0-Back were performed at each visit. During the 2 -Back task, participants viewed stimuli of uppercase letters one at a time on the screen with a crosshair (+) as an inter-stimulus interval between stimuli (see Figure 2 for example). The stimulus appeared for $1 \mathrm{~s}$, and the cross hair for $3 \mathrm{~s}$, providing a $4 \mathrm{~s}$ window to make a response. Participants were instructed to press a button with their right index finger when the current letter matched the letter that appeared two trials 


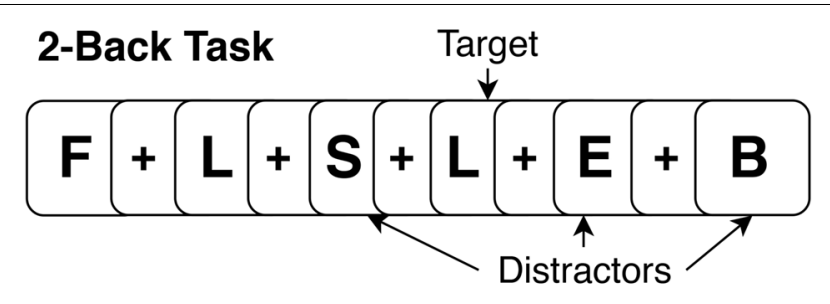

FIGURE 2 | Example of a 2-Back version of N-Back.

back, and press a different button with their right middle finger when stimuli did not match the 2 -Back pattern. The 0 -Back was identical to the 2-Back task; however, it lacked the 2-Back pattern component and was used as an attention-control task. For 0Back, participants were instructed to press a button with their index finger only for the letter " $\mathrm{X}$ " and press a different button with their middle finger for any other letter. As this study aimed to mechanistically evaluate the acute impact of tDCS on working memory networks, participants were trained thoroughly on the $\mathrm{N}$-Back task (2- and 0-Back) outside of the scanner to facilitate stabilization of test-retest reliability and comprehension of the task. The intent of this procedure was to facilitate stability of the activation pattern in the working memory network across subsequent runs and between MRI sessions, and isolate detected change in connectivity due to influences from tDCS alone, rather than from the combination of tDCS and test-retest learning. The training consisted of separate 0 -Back and 2-Back practice sessions with immediate performance feedback. Afterwards, participants performed an identical version of the task as it appeared in scanner without any feedback. The training took approximately $20 \mathrm{~min}$ and was performed before the MRI scan at both active and sham visits. Participants were reminded of instructions while inside the scanner prior to starting the task. N-Back accuracy was analyzed as a percent accuracy score on the 2-Back and 0-Back task. Reaction time was first log-transformed to achieve a normal distribution and the average was taken for each task. Performance (accuracy and reaction time) was evaluated using repeated measures analysis of variance (ANOVA) with stimulation condition (active vs. sham) and time (baseline, during, post) as factors in the model.

\section{Neuroimaging Pre-processing}

Spatial and functional pre-processing was performed using the CONN Toolbox v.2017. $\mathrm{f}^{1}$ and SPM12 ${ }^{2}$ running in MATLAB 2015b (The Mathworks Inc, Natick, MA, USA). Pre-processing steps were used to segment high-resolution T1-weighted anatomical volumes for each participant into gray matter, white matter, and cerebrospinal fluid, and normalize to Montreal Neurological Institute (MNI) space. Functional volumes underwent realignment, slice-timing correction, and normalization to MNI space using the normalized EPI template image in CONN and a spatial Gaussian smoothing kernel of $8 \mathrm{~mm}$. The artifact detection toolbox (ART) was applied to detect any motion artifacts. Motion parameters from the

\footnotetext{
${ }^{1}$ https://www.nitrc.org/projects/conn

${ }^{2}$ https://www.fil.ion.ucl.ac.uk/spm/
}

realignment process were evaluated after pre-processing to identify outliers. Any volumes exceeded a threshold of $>3 \mathrm{~mm}$ (translation) and $>1^{\circ}$ rotation were removed from the analysis. Participants included in the analysis did not exceed thresholds for movement. BOLD data was filtered at a bandpass of 0.008Inf $\mathrm{Hz}$ to reduce low-frequency drift and noise effects. Noise correction was performed using the anatomical componentbased noise correction (aCompCor) method (Behzadi et al., 2007) implemented in the CONN Toolbox and SPM12. This method extracted principal components from white matter and CSF time series, which were then added as confounds in the denoising step within the CONN Toolbox (Behzadi et al., 2007; Whitfield-Gabrieli and Nieto-Castanon, 2012; Demirakca et al., 2016). This step was used to reduce any physiological and subject movement effects from the time series of interest, and enhance sensitivity, specificity, and validity for subsequent functional connectivity analyses (Behzadi et al., 2007; WhitfieldGabrieli and Nieto-Castanon, 2012; Demirakca et al., 2016; Fallon et al., 2016).

\section{Seed-to-Target Regions of Interest (ROIs) Analyses}

Using the BOLD signal, we implemented the CONN Toolbox to estimate measures of functional connectivity (WhitfieldGabrieli et al., 2009; Wei et al., 2017). Previously, the Owen et al. (2005) meta-analysis identified significant activated brain regions during the fMRI N-Back task. We selected the 15 seed regions of interest from this article (Owen et al., 2005) a priori to represent the working memory network in our analyses. Spherical regions of interest (ROIs) were created using the WFU PickAtlas GUI in SPM12 (Maldjian et al., 2003, 2004) and the MNI-coordinates from Owen et al. (2005; Table 1); each ROI was created using the volume $\left(\mathrm{mm}^{3}\right)$ reported in the meta-analysis. The default setting of $10 \mathrm{~mm}$ radius was used if no volume was reported. It should be noted that the Owen et al. (2005) meta-analyses contained overlap between a subset of ROIs. In each case, radius size varied between identified ROIs. While selecting a single representative ROI would reduce the number of multiple comparisons, potentially increasing the number of significant findings in the current study, we chose a conservative approach aimed at maintaining consistency with the Owen et al. (2005) meta-analysis ROIs. In the case of overlap between left frontal pole and one of the two left dorsolateral prefrontal ROIs, the inclusion of these two overlapping ROIs also provides a degree of ability to examine whether tDCS effects within the frontal pole are related to the portion of the frontal pole consistent with DLPFC or the larger extent of the frontal pole ROI. After pre-processing, denoising, and first level analysis (generalized psychophysiological interaction-gPPI; bivariate regression), second level models for the 2-Back task were analyzed for each of the 15 ROIs with combinations of stimulation session (active or sham) and time (baseline, during, post) as conditions in each model. Target regions only included the spherical ROIs to reduce the number of comparisons and focus on nodes within the working memory network. Model contrasts were created within a single stimulation session (e.g., during-active $>$ baseline- 
active) for each seed to all targets. Contrasts with significant connectivity changes (FDR $<0.05$ ) were further analyzed between active vs. sham sessions via a post hoc paired sample $t$-test to quantify the differences between sessions (e.g., duringactive $>$ baseline-active vs. during-sham $>$ baseline-sham). Ttests were FDR 0.05 corrected for multiple comparisons. The 0 -Back was analyzed in an identical manner.

\section{Analytical Approach}

Using the CONN Toolbox, we examined ROI-to-ROI seed-totarget functional connectivity for each seed to all other specified targets in the working memory network via bivariate regression (gPPI) for 2-Back and 0-Back separately. The N-Back blocks in each run were synchronized with the functional data to only capture the task period and not rest or instruction periods. Fisher-transformed bivariate regression coefficients (beta values) between two ROI BOLD time-series were used to identify significant increases or decreases in functional connectivity between the seed-to-target.

\section{RESULTS}

\section{Functional Connectivity During 2-Back}

We examined the effects of active vs. sham stimulation (at two separate visits) across three time points (baseline-active/sham, during-active/sham, and post-active/sham) during the 2-Back working memory task. Seed-to-target analyses demonstrated selective modulation of functional connectivity within the working memory network on the 2-Back task during-active stimulation as shown in Figures 3, 4 and Table 2.

\section{During $>$ Baseline}

\section{Active}

A significant increase in functional connectivity was observed during-active stimulation compared to baseline-active when seeding in the left VLPFC targeting left DLPFC (P-FDR $=0.045)$. On an individual subject level, we observed that $13 / 16(81 \%)$ participants evidenced increases in connectivity.

TABLE 1 | Regions of interest (ROIs), Montreal Neurological Institute (MNI) coordinates, radius for spherical ROls.

\begin{tabular}{lcccc}
\hline Region & $\boldsymbol{x}$ & $\boldsymbol{y}$ & $\boldsymbol{z}$ & Radius \\
\hline LH DLPFC & -37.75 & 50.19 & 13.6 & 6.2 \\
& -46.26 & 22.71 & 18.6 & 14.3 \\
LH frontal pole & -37.75 & 50.19 & 13.6 & 7.5 \\
LH inferior parietal lobule & -37.09 & -47.7 & 45.58 & 10 \\
LH lateral premotor & -26.32 & 6.75 & 53.46 & 9 \\
& -45.96 & 3.1 & 38.47 & 10 \\
LH ventrolateral PFC & -31.36 & 21.11 & 0.58 & 10 \\
Medial cerebellum & 3.12 & -69.09 & -24.69 & 3 \\
RH DLPFC & 44.53 & 38.76 & 24.43 & 12.5 \\
RH inferior Parietal Lobule & 44.97 & -45.49 & 41.73 & 12.46 \\
RH lateral premotor & 31.96 & 11.01 & 49.8 & 15.83 \\
& 31.96 & 11.01 & 49.8 & 10 \\
RH medial posterior parietal & 12.77 & -63.71 & 55.28 & 14.8 \\
RH ventrolateral PFC & 35.58 & 23.26 & -3.01 & 10 \\
Supplementary motor area & -0.588 & 18.57 & 40.65 & 10 \\
\hline
\end{tabular}

$L H$, left hemisphere; $R H$, right hemisphere.

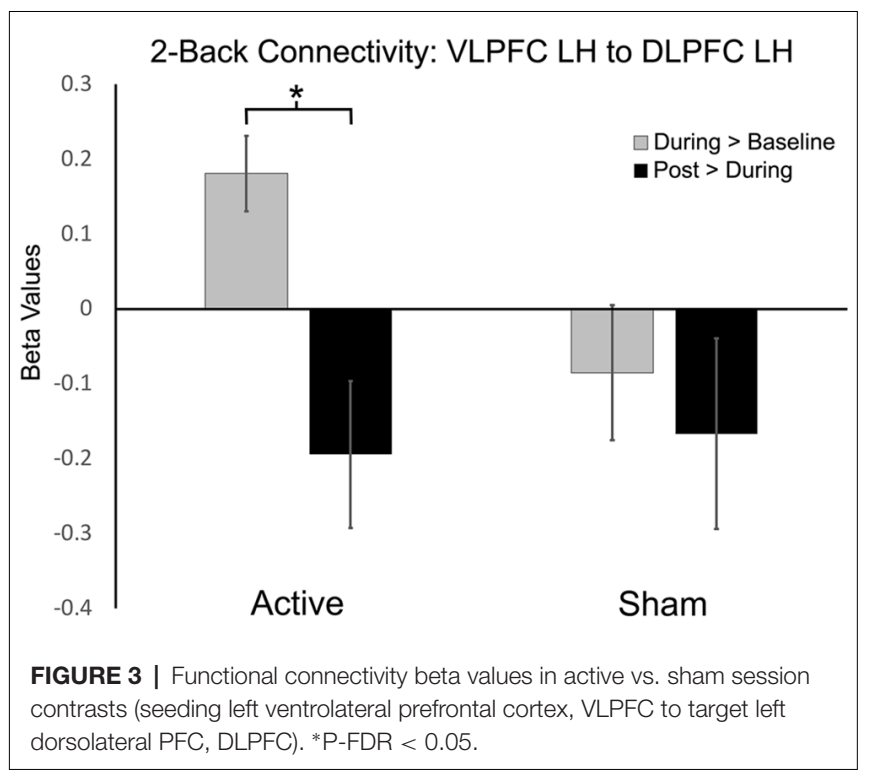

\section{Sham}

We found significantly decreased connectivity during-sham stimulation compared to baseline-sham when seeding in the left lateral premotor targeting the left frontal pole $(\mathrm{P}-\mathrm{FDR}=0.042)$ and the left DLPFC $($ P-FDR $=0.042)$.

\section{Active vs. Sham}

Left ventrolateral PFC (VLPFC) to Left DLPFC: post hoc analyses comparing during-active $>$ baseline-active to during-sham $>$ baseline-sham demonstrated significantly greater connectivity values in the active condition $(t=2.65$, $d f=15, p=0.01, \mathrm{P}-\mathrm{FDR}=0.018$ ).

Left Lateral Premotor to Left Frontal Pole: post hoc analyses comparing during-active $>$ baseline-active to during-sham $>$ baseline-sham demonstrated significantly lower connectivity values in the sham condition $(t=2.77$, $d f=15, p=0.01, \mathrm{P}-\mathrm{FDR}=0.047)$.

Left Lateral Premotor to Left DLPFC: post hoc analyses comparing during-active $>$ baseline-active to during-sham $>$ baseline-sham demonstrated significantly lower connectivity values in the sham condition $(t=2.68$, $d f=15, p=0.01, \mathrm{P}-\mathrm{FDR}=0.047)$.

\section{Post $>$ During}

\section{Active}

There was a significant decrease in connectivity between left DLPFC targeting left VLPFC post-active compared to during-active stimulation $(\mathrm{P}-\mathrm{FDR}=0.049)$. In addition, connectivity between right inferior parietal lobule (seed) and left lateral premotor (target) was also significantly decreased $(\mathrm{P}-\mathrm{FDR}=0.027)$.

\section{Sham}

No significant changes in connectivity were found when comparing post-sham to during-sham stimulation (P-FDR > 0.05). 


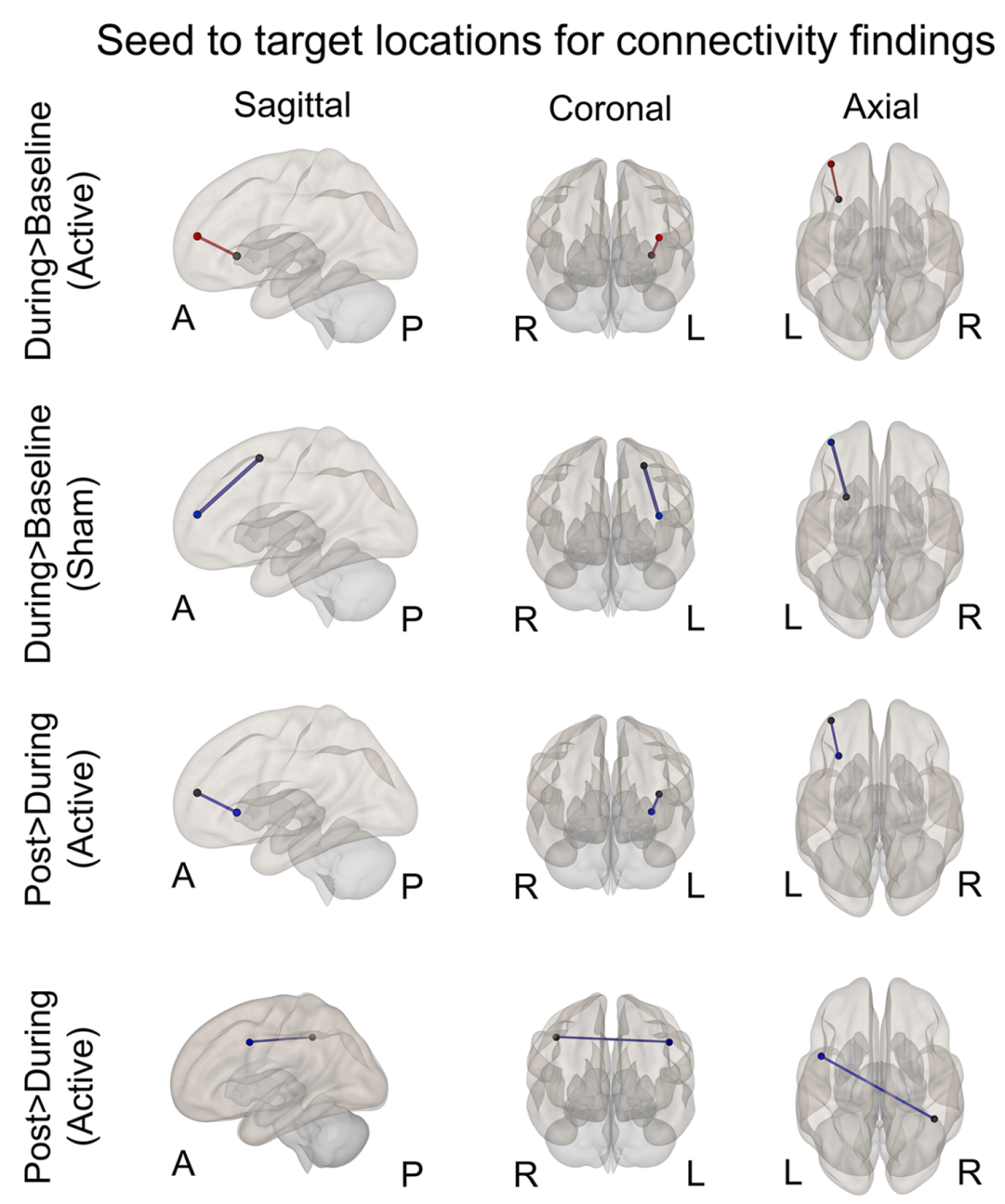

FIGURE 4 | Seed to target locations for significant connectivity findings. See Table 2 for seed to target location names. Nodes colored in black represent the seed location. Nodes color coded in red or blue indicate the target location. Red signifies significantly increased connectivity (FDR $<0.05$ ). Blue signifies significantly decreased connectivity (FDR < 0.05). A, anterior; P, posterior; R, right; L, left.

TABLE 2 | Significant seed to target connectivity values and test statistics for each contrast.

\begin{tabular}{|c|c|c|c|c|c|c|c|c|}
\hline Contrast & Condition & Seed & Target & Beta & $\mathbf{T}_{(15)}$ & P-Unc & P-FDR & Outcome \\
\hline During $>$ Baseline & Active & VLPFC LH & DLPFC LH & 0.18 & 3.59 & 0.002 & $0.045^{*}$ & Increased connectivity \\
\hline During $>$ Baseline & Sham & Lateral premotor LH & Frontal pole LH & -0.32 & -3.4 & 0.003 & $0.042^{*}$ & Decreased connectivity \\
\hline During $>$ Baseline & Sham & Lateral premotor LH & DLPFC LH & -0.38 & -3.28 & 0.005 & $0.042^{*}$ & Decreased connectivity \\
\hline Post $>$ During & Active & DLPFC LH & VLPFC LH & -0.06 & -3.55 & 0.002 & $0.049^{*}$ & Decreased connectivity \\
\hline Post $>$ During & Active & IPL RH & Lateral premotor LH & -0.17 & -3.84 & 0.001 & $0.027^{*}$ & Decreased connectivity \\
\hline
\end{tabular}

${ }^{*} p-F D R<0.05$.

\section{Active vs. Sham}

Left DLPFC to Left VLPFC: post hoc analyses comparing post-active $>$ during-active to post-sham $>$ during-sham did not demonstrate a significant difference between sham and active conditions (P-FDR > 0.05).

Right IPL to Left lateral premotor: analyses comparing post-active $>$ during-active to post-sham $>$ during-sham demonstrated that connectivity values were significantly lower in the active condition $(t=-2.56, d f=15, p=0.021$, $\mathrm{P}-\mathrm{FDR}=0.048)$.

\section{Post $>$ Baseline}

\section{Active}

No significant changes in connectivity were found when comparing post-active stimulation to baseline-active stimulation (P-FDR > 0.05).

\section{Sham}

No significant changes in connectivity were found when comparing post-sham stimulation to baseline-sham $(\mathrm{P}-\mathrm{FDR}>0.05)$. 


\section{Control Contrast: Baseline-Active vs. Baseline-Sham}

No significant changes in connectivity were found between baseline-active vs. baseline-sham stimulation (P-FDR > 0.05).

\section{Functional Connectivity During 0-Back}

The effects of active vs. sham stimulation were evaluated across the three time points during the 0-Back task in an identical manner as 2-Back. Seed-to-target analyses were performed seeding in each ROI targeting all other ROIs in the network. No significant connectivity changes were identified during 0 -Back (P-FDR > 0.05). In addition, there were no significant connectivity differences between baseline-active vs. baseline-sham (P-FDR > 0.05).

\section{N-Back Behavior}

Percent accuracy and reaction time on the N-Back task did not significantly differ between active and sham stimulation conditions at the various time points $(p>0.05)$ as reported in Table 3. Evaluation of linear and non-linear fits for performance data demonstrated a trend towards faster reaction time for 2-Back targets (tests of within-subject contrast fit with quadratic equation; $F=2.146, d f=1.15$, partial eta squared $=0.125$, $p=0.16$ ) and improved 2-Back target accuracy (fit with linear equation; $F=3.199, d f=1.15$, partial eta squared $=0.176$, $p=0.09)$ during active-stimulation using repeated measures ANOVA within-subject contrast (time $\times$ stimulation condition). Nonetheless, performance was not significantly different for accuracy and reaction time.

\section{DISCUSSION}

To the best of our knowledge, this is the first study to investigate the mechanistic acute and after-effects of bilateral frontal tDCS on functional connectivity of the working memory network in older adults. Our results demonstrate the ability of in-scanner tDCS paired with an N-Back task to acutely modulate functional connectivity in the working memory network. We found increased functional connectivity within the working memory network (seeding left VLPFC targeting left DLPFC) while participants performed the 2-Back task during active stimulation compared to baseline. Moreover, the post hoc between-condition analysis demonstrated significantly greater connectivity values in the active condition $(\mathrm{P}-\mathrm{FDR}=0.018)$. In contrast, during sham stimulation compared to baseline, we observed a significant decrease in functional connectivity during the 2-Back task (seeding left lateral premotor to left frontal pole and left DLPFC). Importantly, the changes in functional connectivity that occurred during active stimulation only occurred while performing the more challenging 2-Back task, and not during the less challenging 0-Back task. Additionally, increased left frontal connectivity during active stimulation trended towards decreasing post-stimulation, and may suggest that functional connectivity returned to baseline values after stimulation was stopped. This is also potentially supported by a significant decrease in connectivity that was observed at post-active stimulation compared to during active stimulation when seeding left DLPFC targeting left VLPFC. Collectively, these data suggest the ability of acute bilateral F3/F4 tDCS at $2 \mathrm{~mA}$ to selectively modulate frontal functional connectivity of working memory related brain regions in older adults during stimulation. These results provide important insight into: (1) selective effects of tDCS on functional connectivity; (2) state-dependent effects of tDCS; (3) the importance of timing tDCS delivery; and (4) effects of $2 \mathrm{~mA}$ stimulation under the cathode electrode.

Our results demonstrated an increase in connectivity between two frontal lobe structures (VLPFC and DLPFC) during-active tDCS. Sub-regions of the frontal lobe are essential for manipulation of verbal and spatial knowledge (Barbey et al., 2013). Functionally, the VLPFC has exhibited enhanced activity during various cognitive control processes such as selection, maintenance, and retrieval of goal directed information. The DLPFC has shown critical involvements during task delay periods and facilitates the ability to keep task-relevant information active, in addition to manipulating that information to accomplish the task. Together, the DLPFC and VLPFC demonstrate enhanced activity in cognitive control processes such as monitoring, manipulating, or processing goal directed information, all processes vital to working memory (D’Esposito et al., 1999; Petrides, 2000; Blumenfeld et al., 2013).

Our finding of functional connectivity increase duringactive stimulation on 2-Back, and not 0 -Back is consistent with previous investigations of state-dependent effects of tDCS. Gill et al. (2014) provided evidence that cognitive enhancement capacity of tDCS may depend on the nature of the task being performed during stimulation (Gill et al., 2014). A challenging and more cognitively demanding 3-Back task produced greater proficiency on a separate working memory task (Paced Auditory Serial Addition Task, PASAT). In contrast, the less cognitively demanding 1-Back task performed during stimulation did not result in an improvement on the PASAT. Our results support prior research that the effects of tDCS are state dependent on more cognitively demanding tasks (2-Back) vs. less demanding tasks (0-Back).

Previous studies examining tDCS paired with cognitive training indicate the importance of timing of stimulation. Martin et al. $(2013,2014)$ demonstrated that gains from tDCS were

TABLE 3 | Mean percent task accuracy and standard error on 2-Back and 0-Back for each condition; mean reaction time and standard error in milliseconds for each condition.

\begin{tabular}{|c|c|c|c|c|c|c|}
\hline N-Back performance (mean, std. error) & Baseline-Active & During-Active & Post-Active & Baseline-Sham & During-Sham & Post-Sham \\
\hline 2-Back accuracy & $84 \%(4 \%)$ & $81 \%(5 \%)$ & $82 \%(6 \%)$ & $85 \%(4 \%)$ & $85 \%(3 \%)$ & $79 \%(4 \%)$ \\
\hline 0-Back accuracy & $89 \%(3 \%)$ & $92 \%(4 \%)$ & $84 \%(6 \%)$ & $89 \%(4 \%)$ & $89 \%(3 \%)$ & $88 \%(3 \%)$ \\
\hline 2-Back reaction time & $959.3(57.2)$ & $847.2(63.6)$ & $868.5(39.0)$ & $1015.2(64.5)$ & $955.5(62.5)$ & $886.6(51.4)$ \\
\hline 0-Back reaction time & $791.2(55.1)$ & $738.5(60.6)$ & $756.5(55.5)$ & $830.3(74.6)$ & $760.3(62.7)$ & $739.6(56.9)$ \\
\hline
\end{tabular}


significantly greater when stimulation was delivered during an adaptive $\mathrm{N}$-Back cognitive training task vs. stimulation delivered prior to training (Martin et al., 2013, 2014). These data suggest that optimal gains from tDCS may be achieved by "co-stimulating" neural networks through both behavior and electrical stimulation. Our finding of increased functional connectivity during active stimulation, and not at baseline or post stimulation, appears to be consistent with this notion, and further highlights the importance of timing and delivery of tDCS for optimizing brain-based effects. While absence of post-stimulation after effects on functional connectivity do not preclude behavioral effects of $\mathrm{tDCS}$, these data might suggest that there is an optimal window for leveraging brain-based changes of tDCS during the period of stimulation. However, it is important to use caution when interpreting our results in this domain, as our participants only demonstrated trends for improved accuracy and reaction time in the current study.

Previous studies in tDCS and the motor cortex have shown that $1 \mathrm{~mA}$ vs. $2 \mathrm{~mA}$ of intensity can have differing physiological responses under the anode vs. cathode electrodes by measuring changes in motor evoked potentials (MEPs). At $1 \mathrm{~mA}$, an increase in excitability is typically seen under the anode electrode, whereas the cathode electrode demonstrates a decrease in excitability (Bindman et al., 1964; Nitsche and Paulus, 2000; Nitsche et al., 2003a,b). Batsikadze et al. (2013) demonstrated that $2 \mathrm{~mA}$ tDCS can produce a net increase in excitability under both the anode and cathode electrodes (Batsikadze et al., 2013). At present, there are no MEP-like markers within the frontal lobes that indicate physiological change induced via tDCS. However, our finding of increased functional connectivity of left frontal regions during active stimulation may support prior motor cortex findings of increased excitability under the cathode electrode at $2 \mathrm{~mA}$ in frontal cortices. If the cathode electrode induced a decrease in excitability in underlying tissue, we might expect to see decreased connectivity, or no change, yet we saw an increase in functional connectivity lateralized to the left hemisphere where the cathode electrode was placed. Therefore, these data appear, at least in part, to support a growing body of research suggesting that $2 \mathrm{~mA}$ tDCS may result in increased excitability under the cathode electrode.

When interpreting the results, limitations of the study should be considered. Artifacts in the MRI environment could alter imaging data; we took into consideration how introducing current into the MRI may cause artifacts. Our task paradigm randomized and switched between 2- and 0-Back for duringactive stimulation. We did not identify any significant changes in functional connectivity while 0 -Back was performed in duringactive stimulation. The lack of change in during-active vs. during-sham stimulation on the 0-Back task provides further evidence that the observed connectivity increase was not caused by potential artifacts directly induced by current delivery. This suggests the connectivity changes that occurred during active stimulation on the 2-Back were a result of stimulation. This study did not demonstrate statistically significant behavioral improvements in the active vs. sham session, although a trend for significance and medium effect sizes were present. Our study trained participants prior to in-scanner performance to stabilize the behavioral and network performance across multiple runs, to better enable direct evaluation of acute impact of delivered current flow on functional connectivity in working memory networks. As expected, stable and high levels of performance likely minimized our ability to detect large changes in N-back performance. In addition, limited power due to small sample size likely played a role in these results. It is also important to consider the range of cognitive abilities of the selected sample. The current study used a liberal MoCA cutoff of 20, with an average of 26.5. Thus, the older adult sample in the current study included not only participants that could be classified as "normal healthy" older adults, but also some participants that may not be entirely cognitive normal. Nonetheless, participants were excluded for diagnosis of mild cognitive impairment or dementia. There are also nuances to performing fMRI in older adults that must be considered. The process of aging on the brain may uniquely impact adaptive network mechanisms. Thus, effects found in the current study may not extend to younger adults. Future studies replicated the current findings in younger adults would help to expand our understanding of tDCS mechanisms across the lifespan. As the population studied involves older adults, 25\% or more of participants may have beta-amyloid present in the brain. As beta-amyloid can impact functional connectivity, this factor may play a role in our overall results and further stresses the need for follow-up studies in young adults and older adults with corresponding beta-amyloid positron emission tomography.

\section{CONCLUSION}

The underlying mechanism(s) of action of tDCS and its effects on brain function are not yet fully understood. Our results demonstrated that acute bilateral frontal tDCS selectively modulates functional brain connectivity in the working memory network of older adults. These data indicate the importance of timing of stimulation and the critical influence that state/cognitive effort may have during the period of stimulation. Further research pairing tDCS with unimodal and multimodal neuroimaging is needed to continue elucidating the mechanism(s) of tDCS brain-based effects on cognition.

\section{DATA AVAILABILITY}

The datasets generated for this study are available on request to the corresponding author.

\section{AUTHOR CONTRIBUTIONS}

NN, AO'S, AI, RT, LR, EP, RC and AW contributed to the manuscript. NN and AW performed data analyses. All authors approved the final version of the manuscript.

\section{FUNDING}

This work was supported in part by the National Institute of Aging/National Institutes of Health (K01AG050707, R01AG054077), the Evelyn F. McKnight Brain Research Foundation, and the University of Florida Center for Cognitive Aging and Memory. 


\section{REFERENCES}

Andrews-Hanna, J. R., Snyder, A. Z., Vincent, J. L., Lustig, C., Head, D., Raichle, M. E., et al. (2007). Disruption of large-scale brain systems in advanced aging. Neuron 56, 924-935. doi: 10.1016/j.neuron.2007.10.038

Baddeley, A. (1992). Working memory. Science 255, 556-559. doi: 10.1126/science. 1736359

Baddeley, A. (2003). Working memory: looking back and looking forward. Nat. Rev. Neurosci. 4, 829-839. doi: 10.1038/nrn1201

Barbey, A. K., Koenigs, M., and Grafman, J. (2013). Dorsolateral prefrontal contributions to human working memory. Cortex 49, 1195-1205. doi: 10.1016/j.cortex.2012.05.022

Batsikadze, G., Moliadze, V., Paulus, W., Kuo, M.-F., and Nitsche, M. A. (2013). Partially non-linear stimulation intensity-dependent effects of direct current stimulation on motor cortex excitability in humans. J. Physiol. 591, 1987-2000. doi: 10.1113/jphysiol.2012.249730

Behzadi, Y., Restom, K., Liau, J., and Liu, T. T. (2007). A component based noise correction method (CompCor) for BOLD and perfusion based FMRI. Neuroimage 37, 90-101. doi: 10.1016/j.neuroimage.2007.04.042

Bikson, M., Brunoni, A. R., Charvet, L. E., Clark, V. P., Cohen, L. G., Deng, Z.-D., et al. (2018). Rigor and reproducibility in research with transcranial electrical stimulation: an NIMH-sponsored workshop. Brain Stimul. 11, 465-480. doi: 10.1016/j.brs.2017.12.008

Bikson, M., Grossman, P., Thomas, C., Zannou, A. L., Jiang, J., Adnan, T., et al. (2016). Safety of transcranial direct current stimulation: evidence based update 2016. Brain Stimul. 9, 641-661. doi: 10.1016/j.brs.2016.06.004

Bindman, L. J., Lippold, O. C., and Redfearn, J. W. (1964). The action of brief polarizing currents on the cerebral cortex of the rat (1) during current flow and (2) in the production of long-lasting after-effects. J. Physiol. 172, 369-382. doi: 10.1113/jphysiol.1964.sp007425

Blumenfeld, R. S., Nomura, E. M., Gratton, C., and D’Esposito, M. (2013). Lateral prefrontal cortex is organized into parallel dorsal and ventral streams along the rostro-caudal axis. Cereb. Cortex 23, 2457-2466. doi: 10.1093/cercor/bhs223

Boisgueheneuc, F. D., Levy, R., Volle, E., Seassau, M., Duffau, H., Kinkingnehun, S., et al. (2006). Functions of the left superior frontal gyrus in humans: a lesion study. Brain 129, 3315-3328. doi: 10.1093/brain/awl244

Cabeza, R., Anderson, N. D., Locantore, J. K., and McIntosh, A. R. (2002). Aging gracefully: compensatory brain activity in high-performing older adults. Neuroimage 17, 1394-1402. doi: 10.1006/nimg.2002.1280

D’Esposito, M., Postle, B. R., Ballard, D., and Lease, J. (1999). Maintenance versus manipulation of information held in working memory: an event-related FMRI study. Brain Cogn. 41, 66-86. doi: 10.1006/brcg.1999.1096

Demirakca, T., Cardinale, V., Dehn, S., Ruf, M., and Ende, G. (2016). The exercising brain-changes in functional connectivity induced by an integrated multimodal cognitive and whole body motor coordination training. Neural Plast. 2016:8240894. doi: 10.1155/2016/8240894

Fallon, N., Chiu, Y., Nurmikko, T., and Stancak, A. (2016). Functional connectivity with the default mode network is altered in fibromyalgia patients. PLoS One 11:e0159198. doi: 10.1371/journal.pone.0159198

Gazzaley, A., Sheridan, M. A., Cooney, J. W., and D’Esposito, M. (2007). Agerelated deficits in component processes of working memory. Neuropsychology 21, 532-539. doi: 10.1037/0894-4105.21.5.532

Gill, J., Shah-Basak, P. P., and Hamilton, R. (2014). It's the thought that counts: examining the task-dependent effects of transcranial direct current stimulation on executive function. Brain Stimul. 8, 253-259. doi: 10.1016/j.brs.2014.10.018

Goldman-Rakic, P. S. (1987). "Circuitry of primate prefrontal cortex and regulation of behavior by representational memory-comprehensive physiology," in Handbook of Physiology, ed. E. Goldberg (New York, NY: John Wiley \& Sons), 373-417.

Goldman-Rakic, P. S. (1996). Regional and cellular fractionation of working memory. Proc. Natl. Acad. Sci. U S A 93, 13473-13480. doi: 10.1073/pnas.93. 24.13473

Gomes-Osman, J., Indahlastari, A., Fried, P. J., Cabral, D. L. F., Rice, J., Nissim, N. R., et al. (2018). Non-invasive brain stimulation: probing intracortical circuits and improving cognition in the aging brain. Front. Aging Neurosci. 10:177. doi: 10.3389/fnagi.2018.00177

Hampson, M., Driesen, N. R., Skudlarski, P., Gore, J. C., and Constable, R. T. (2006). Brain connectivity related to working memory performance.
J. Neurosci. 26, 13338-13343. doi: 10.1523/JNEUROSCI.3408 06.2006

Hampson, M., Driesen, N. R., Roth, J. K., Gore, J. C., and Constable, R. T. (2010) Functional connectivity between task-positive and task-negative brain areas and its relation to working memory performance. Magn. Reson. Imaging 28, 1051-1057. doi: 10.1016/j.mri.2010.03.021

Jones, K. T., Stephens, J. A., Alam, M., Bikson, M., and Berryhill, M. E. (2015). Longitudinal neurostimulation in older adults improves working memory. PLoS One 10:e0121904. doi: 10.1371/journal.pone.0121904

Keller, J. B., Hedden, T., Thompson, T. W., Anteraper, S. A., Gabrieli, J. D. E., and Whitfield-Gabrieli, S. (2015). Resting-state anticorrelations between medial and lateral prefrontal cortex: association with working memory, aging, and individual differences. Cortex 64, 271-280. doi: 10.1016/j.cortex.2014.12.001

Khadka, N., Woods, A. J., and Bikson, M. (2019). "Transcranial direct current stimulation electrodes," in Practical Guide to Transcranial Direct Current Stimulation, eds H. Knotkova, M. Nitsche, M. Bikson and A. Woods (Cham: Springer International Publishing), 263-291.

Knotkova, H., Nitsche, M., Bikson, M., and Woods, A. J. (2019). "Practical guide to transcranial direct current stimulation," in Principles, Procedures, and Applications, 1st Edn. eds H. Knotkova, M. Nitsche, M. Bikson and A. J. Woods (Switzerland, AG: Springer Nature), 1-651.

Li, S.-C., Lindenberger, U., and Sikström, S. (2001). Aging cognition: from neuromodulation to representation. Trends Cogn. Sci. 5, 479-486. doi: 10.1016/s1364-6613(00)01769-1

Maldjian, J. A., Laurienti, P. J., and Burdette, J. H. (2004). Precentral gyrus discrepancy in electronic versions of the talairach atlas. Neuroimage 21, 450-455. doi: 10.1016/j.neuroimage.2003.09.032

Maldjian, J. A., Laurienti, P. J., Kraft, R. A., and Burdette, J. H. (2003). An automated method for neuroanatomic and cytoarchitectonic atlasbased interrogation of FMRI data sets. Neuroimage 19, 1233-1239. doi: 10.1016/s1053-8119(03)00169-1

Martin, D. M., Liu, R., Alonzo, A., Green, M., and Loo, C. K. (2014). Use of transcranial direct current stimulation (TDCS) to enhance cognitive training: effect of timing of stimulation. Exp. Brain Res. 232, 3345-3351. doi: 10.1007/s00221-014-4022-x

Martin, D. M., Liu, R., Alonzo, A., Green, M., Player, M. J., Sachdev, P., et al. (2013). Can transcranial direct current stimulation enhance outcomes from cognitive training? A randomized controlled trial in healthy participants. Int J. Neuropsychopharmacol. 16, 1927-1936. doi: 10.1017/s1461145713000539

McLaren, M. E., Nissim, N. R., and Woods, A. J. (2018). The effects of medication use in transcranial direct current stimulation: a brief review. Brain Stimul. 11, 52-58. doi: 10.1016/j.brs.2017.10.006

Mograbi, D. C., Assis Faria, C., Fichman, H. C., Paradela, E. M., and Alves Lourenço, R. (2014). Relationship between activities of daily living and cognitive ability in a sample of older adults with heterogeneous educational level. Ann. Indian Acad. Neurol. 17, 71-76. doi: 10.4103/0972-2327.128558

Monte-Silva, K., Kuo Fang, M., Hessenthaler, S., Fresnoza, S., Liebetanz, D., Paulus, W., et al. (2013). Induction of late LTP-like plasticity in the human motor cortex by repeated non-invasive brain stimulation. Brain Stimul. 6, 424-432. doi: 10.1016/j.brs.2012.04.011

Nissim, N. R., O’Shea, A. M., Bryant, V., Porges, E. C., Cohen, R., and Woods, A. J. (2017). Frontal structural neural correlates of working memory performance in older adults. Front. Aging Neurosci. 8:328. doi: 10.3389/fnagi.2016.00328

Nitsche, M. A., Fricke, K., Henschke, U., Schlitterlau, A., Liebetanz, D., Lang, N., et al. (2003a). Pharmacological modulation of cortical excitability shifts induced by transcranial direct current stimulation in humans. J. Physiol. 553, 293-301. doi: 10.1113/jphysiol.2003.049916

Nitsche, M. A., Nitsche, M. S., Klein, C. C., Tergau, F., Rothwell, J. C., and Paulus, W. (2003b). Level of action of cathodal DC polarisation induced inhibition of the human motor cortex. Clin. Neurophysiol. 114, 600-604. doi: 10.1016/s1388-2457(02)00412-1

Nitsche, M. A., Liebetanz, D., Schlitterlau, A., Henschke, U., Fricke, K., Frommann, K., et al. (2004). GABAergic modulation of dc stimulation-induced motor cortex excitability shifts in humans. Eur. J. Neurosci. 19, 2720-2726. doi: 10.1111/j.0953-816x.2004.03398.x

Nitsche, M. A., and Paulus, W. (2000). Excitability changes induced in the human motor cortex by weak transcranial direct current stimulation. J. Physiol. 527, 633-639. doi: 10.1111/j.1469-7793.2000.t01-1-00633.x 
Owen, A. M., McMillan, K. M., Laird, A. R., and Bullmore, E. (2005). Nback working memory paradigm: a meta-analysis of normative functional neuroimaging studies. Hum. Brain Mapp. 25, 46-59. doi: 10.1002/hbm.20131

Pelletier, S. J., and Cicchetti, F. (2015). Cellular and molecular mechanisms of action of transcranial direct current stimulation: evidence from in vitro and in vivo models. Int. J. Neuropsychopharmacol. 18:pyu047. doi: 10.1093/ijnp/pyu047

Petrides, M. (2000). Dissociable roles of mid-dorsolateral prefrontal and anterior inferotemporal cortex in visual working memory. J. Neurosci. 20, 7496-7503. doi: 10.1523/jneurosci.20-19-07496.2000

Reato, D., Salvador, R., Bikson, M., Opitz, A., Dmochowski, J., and Miranda, P. C. (2019). "Principles of transcranial direct current stimulation (TDCS): introduction to the biophysics of TDCS," in Practical Guide to Transcranial Direct Current Stimulation, eds H. Knotkova, M. Nitsche, M. Bikson and A. Woods (Cham: Springer International Publishing), 45-80.

Richmond, L. L., Morrison, A. B., Chein, J. M., and Olson, I. R. (2011). Working memory training and transfer in older adults. Psychol. Aging 26, 813-822. doi: 10.1037/a0023631

Richmond, L. L., Wolk, D., Chein, J., and Olson, I. R. (2014). Transcranial direct current stimulation enhances verbal working memory training performance over time and near transfer outcomes. J. Cogn. Neurosci. 26, 2443-2454. doi: 10.1162/jocn_a_00657

Salthouse, T. A., Mitchell, D. R., Skovronek, E., and Babcock, R. L. (1989). Effects of adult age and working memory on reasoning and spatial abilities. J. Exp. Psychol. Learn. Mem. Cogn. 15, 507-516. doi: 10.1037//0278-7393.15.3.507

Stephens, J. A., and Berryhill, M. E. (2016). Older adults improve on everyday tasks after working memory training and neurostimulation. Brain Stimul. 9, 553-559. doi: 10.1016/j.brs.2016.04.001

Wang, M., Gamo, N. J., Yang, Y., Jin, L. E., Wang, X.-J., Laubach, M., et al. (2011). Neuronal basis of age-related working memory decline. Nature 476, 210-213. doi: 10.1038/nature10243

Wei, P., Zhang, Z., Lv, Z., and Jing, B. (2017). Strong functional connectivity among homotopic brain areas is vital for motor control in unilateral limb movement. Front. Hum. Neurosci. 11:366. doi: 10.3389/fnhum.2017.00366
Whitfield-Gabrieli, S., and Nieto-Castanon, A. (2012). Conn: a functional connectivity toolbox for correlated and anticorrelated brain networks. Brain Connect. 2, 125-141. doi: 10.1089/brain.2012.0073

Whitfield-Gabrieli, S., Thermenos, H. W., Milanovic, S., Tsuang, M. T., Faraone, S. V., McCarley, R. W., et al. (2009). Hyperactivity and hyperconnectivity of the default network in schizophrenia and in firstdegree relatives of persons with schizophrenia. Proc. Natl. Acad. Sci. U S A 106, 1279-1284. doi: 10.1073/pnas.0809141106

Woods, A. J., Antal, A., Bikson, M., Boggio, P. S., Brunoni, A. R., Celnik, P., et al. (2016). A technical guide to TDCS and related non-invasive brain stimulation tools. Clin. Neurophysiol. 127, 1031-1048. doi: 10.1016/j.clinph.2015.11.012

Woods, A. J., Antonenko, D., Flöel, A., Hampstead, B. M., Clark, D., and Knotkova, H. (2019a). "Transcranial direct current stimulation in aging research," in Practical Guide to Transcranial Direct Current Stimulation, eds H. Knotkova, M. Nitsche, M. Bikson and A. Woods (Cham: Springer International Publishing), 569-595

Woods, A. J., Bikson, M., Chelette, K., Dmochowski, J., Dutta, A., Esmaeilpour, Z., et al. (2019b). "Transcranial direct current stimulation integration with magnetic resonance imaging, magnetic resonance spectroscopy, near infrared spectroscopy imaging, and electroencephalography," in Practical Guide to Transcranial Direct Current Stimulation, eds H. Knotkova, M. Nitsche, M. Bikson and A. Woods (Cham: Springer International Publishing), 293-345.

Conflict of Interest Statement: The authors declare that the research was conducted in the absence of any commercial or financial relationships that could be construed as a potential conflict of interest.

Copyright (c) 2019 Nissim, O'Shea, Indahlastari, Telles, Richards, Porges, Cohen and Woods. This is an open-access article distributed under the terms of the Creative Commons Attribution License (CC BY). The use, distribution or reproduction in other forums is permitted, provided the original author(s) and the copyright owner(s) are credited and that the original publication in this journal is cited, in accordance with accepted academic practice. No use, distribution or reproduction is permitted which does not comply with these terms. 